Chronic Obstructive Pulmonary Diseases: Journal of the COPD Foundation

\title{
Ambulatory Oxygen for Exercise-Induced Desaturation and Dyspnea in Chronic Obstructive Pulmonary Disease (COPD): Systematic Review and Meta-Analysis
}

Stanley I. Ejiofor, $\mathrm{MRCP}^{1}$ Susan Bayliss, ${ }^{1}$ Abubacarr Gassamma, ${ }^{1}$ Alice M. Turner, MRCP, $\mathrm{PhD}^{1,2}$

\begin{abstract}
Introduction: Ambulatory oxygen therapy is indicated in patients that use long term oxygen therapy (LTOT) and current guidelines suggest its use in patients who exhibit exertional desaturation if there is a demonstrable improvement in exercise capacity. Evidence for this is largely derived from single assessment studies which have shown clear benefit in this setting when oxygen versus air is used. The long term effects, however, of ambulatory oxygen therapy in this particular group of patients is controversial.

Methods: We conducted a systematic review of published literature from 1980 to June 2014 for trials in which ambulatory oxygen was compared to placebo in chronic obstructive pulmonary disease (COPD) patients not on LTOT. We also reviewed the effectiveness of devices delivering ambulatory oxygen. Outcome measures were focused towards exercise capacity, Borg scores and the ability of the delivery devices to maintain oxygen saturations on exercise.
\end{abstract}

Results: Twenty three studies (620 patients) were included in the review. Nine studies evaluated the clinical effectiveness of ambulatory oxygen and 14 studies evaluated the impact of the delivery devices. Ambulatory oxygen had no statistical effect on improving exercise capacity when assessed by the 6-minute walk test (6MWT) or the endurance shuttle walk test (ESWT); $p=0.44$ and $p=0.29$ respectively. End of test Borg scores showed no statistical improvement with ambulatory oxygen therapy during 6MWT $(p=0.68)$. Oxygen conserving devices significantly improved oxygen saturations on exercise compared with continuous flow nasal cannulae $(p=0.04)$.

Conclusion: Ambulatory oxygen therapy has limited long term benefit in improving functional exercise capacity or Borg dyspnea scores.

\begin{abstract}
Abbreviations: long-term oxygen therapy, LTOT; chronic obstructive pulmonary disease, COPD; 6-minute walking test, 6MWT; endurance shuttle walk test, ESWT; exercise-induced desaturation, EID; quality of life, QoL; randomized controlled trials, RCTs; pulmonary rehabilitation, PR; standard deviation, SD; activities of daily living, ADLs; baseline/transitional dyspnea scores, BDI/TDI; blood oxygen saturation, $\mathrm{SpO}_{2}$; Chronic Respiratory Questionnaire, CRQ; Hamilton Depression and Anxiety Scale, HADS; pulmonary function tests, PFTs; minimal clinically important difference, MCID; St. George's Respiratory Questionnaire, SGRQ; work rate, WR; partial pressure of oxygen in arterial blood, $\mathrm{PaO}_{2}$; 5-minute walk test, 5MWT; American Thoracic Society, ATS; demand oxygen devices, DODs; pendant reservoir cannulae, PRC; metabolic equivalents, METS; not reported, NR; confidence interval, CI; oxygen conserving devices, OCDs; continuous flow nasal cannulae, CFNC

Funding Support: No specific, designated funds were received for this study. However, during the term of this study, Dr. Turner received an unrestricted grant from the Linde Real Fund to conduct a clinical trial of ambulatory oxygen.

Date of Acceptance: October 21, 2015

Citation: Ejiofor SI, Bayliss S, Gassamma B, Turner AM. Ambulatory oxygen for exercise-induced desaturation and dyspnea in chronic obstructive pulmonary disease (COPD): systematic review and meta-analysis. Chronic Obstr Pulm Dis (Miami). 2016;3(1):419-434. doi:http://dx.doi.org/10.15326/jcopdf.3.1.2015.0146.
\end{abstract}

\section{This article conatins an online supplement}

1 University of Birmingham, Edgbaston Birmingham, United Kingdom
2 Heart of England NHS Foundation Trust, Birmingham, United Kingdom 


\section{Address correspondence to:}

Alice M. Turner

College of Medical and Dental Sciences

University of Birmingham

QEHB Research Labs

Mindelsohn Way

Birmingham, UK

B15 2WB

01213713886.

Fax 01213713887

Email:a.m.wood@bham.ac.uk

\section{Keywords:}

conserving device; shuttle walk; ambulatory oxygen; exercise-induced dyspnea

\section{Background}

Chronic obstructive pulmonary disease (COPD) is a leading cause of morbidity and mortality, with prevalence anticipated to increase due to persistent exposure to COPD risk factors and an ageing population. ${ }^{1}$ Hypoxaemia and dyspnea are hallmark features; in many individuals gaseous exchange becomes more impaired as the disease progresses, leading to hypoxemia at rest or on exercise alone. Several mechanisms causing this are diffusion limitation, ventilation/perfusion mismatches and shunting. Exercise-induced desaturation (EID) is likely to contribute to exercise limitation in COPD. EID is defined as an arterial oxygen saturation $<88 \%$ (nadir) measured by pulse oximetry during exercise. ${ }^{2}$ Current evidence suggests that EID is common, and may lead to worse quality of life(QoL) and increased mortality. ${ }^{3,4}$

Ambulatory oxygen therapy is defined as the use of supplementary oxygen during activities of daily living. Although the provision of ambulatory oxygen varies widely across countries, its use is generally accepted in patients who exhibit exertional desaturation if there is a demonstrable improvement in exercise capacity or dyspnea scores. 5 "6 While the benefits of oxygen treatment in the form of long term oxygen therapy $(\mathrm{LTOT})^{7,8}$ are well recognized, the long term benefits of ambulatory oxygen in COPD are controversial. A Cochrane review demonstrated benefit of ambulatory oxygen in improving exercise capacity in moderate to severe COPD, albeit mostly in single assessment studies. ${ }^{9}$ In a subsequent review evaluating ambulatory oxygen in the context of pulmonary rehabilitation programs, no benefit was demonstrated. ${ }^{10}$

Current clinical use and associated costs of ambulatory oxygen suggest that assessing longer term benefits of ambulatory oxygen in COPD in a domiciliary and rehabilitation setting is required. Thus the aim of this review was to determine:

1) The clinical effectiveness of long term ambulatory oxygen therapy (defined by more than 6 weeks duration) in patients with COPD exhibiting EID or exertional dyspnea who do not meet the criteria for LTOT;

2) The relative clinical effectiveness of different methods of delivering ambulatory oxygen in COPD patients and the optimum mode of delivery of the intervention;

3) The cost-effectiveness of ambulatory oxygen in COPD patients not on LTOT compared to usual care and the relative cost-effectiveness of different methods of delivering ambulatory oxygen.

\section{Methods}

Studies were eligible for inclusion if they included adult patients (18 years and over) and met the criteria in Table 1: Randomized controlled trials (RCTs), non RCTs and crossover studies were included for clinical and cost effectiveness aims. To address aim 2: ambulatory oxygen delivery device efficacy, single assessment studies and patients who met LTOT criteria were included (for this aim only). The definition of COPD was broadened to include emphysema and chronic bronchitis. The protocol is registered with Prospero (CRD42012002984).

\section{Search Strategy}

To maximize efficiency, 2 search strategies were undertaken, under the guidance of an information search specialist, as outlined in the supplementary methods. The literature search included the following databases; MEDLINE, MEDLINE in Process, EMBASE, Cochrane(CENTRAL), Science Citation Index, National Health Service Economic Evaluation Database, PEDro, Health Technology Assessment database, ClinicalTrials. gov, Cumulative Index to Nursing and Allied Health Literature, and the Cochrane Airways specialized register. Following this, the references of each included study, as well as any review articles found, were searched for additional articles that may contain further studies.

Database searches were conducted between 1980 and June 2013 inclusive as older studies; pre 1980 would have been before the introduction of LTOT. An updated search run to June 2014 yielded no further articles suitable for inclusion. 


\section{Table 1. Eligibility Criteria}

\section{Aim}

Clinical effectiveness

of ambulatory oxygen

\section{Participants}

Stable COPD;

Evidence of exercise

desaturation/exertional

dyspnea;

Not on LTOT;

Follow up longer

than 6 weeks
Intervention Comparisons

Ambient air/

compressed air

\section{Outcomes}

Ambulatory
oxygen

Exercise capacity;

Borg dyspnea scores; QoL

\begin{tabular}{|c|c|c|c|c|}
\hline $\begin{array}{l}\text { Efficacy of ambulatory } \\
\text { oxygen delivery device }\end{array}$ & $\begin{array}{l}\text { Stable COPD with follow } \\
\text { up of any duration }\end{array}$ & \multicolumn{2}{|c|}{$\begin{array}{l}\text { Any form of delivery device for } \\
\text { ambulatory oxygen }\end{array}$} & $\begin{array}{l}\text { Exercise capacity } \\
\text { Exercise } \mathrm{SpO}_{2}\end{array}$ \\
\hline Cost effectiveness & $\begin{array}{l}\text { As per clinical } \\
\text { effectiveness aim }\end{array}$ & $\begin{array}{l}\text { Ambulatory } \\
\text { oxygen }\end{array}$ & Usual care & $\begin{array}{l}\text { QoL costs; } \\
\text { Incremental cost ratios }\end{array}$ \\
\hline
\end{tabular}

LTOT: long term oxygen therapy; QoL: quality of life; $\mathrm{SpO}_{2}$ : blood oxygen saturation

\section{Study Selection, Data Extraction and Risk of Bias}

Two reviewer authors (SE and AT) independently selected trials for inclusion in the review. They first reviewed titles and abstracts and obtained full articles judged as potentially eligible. These were screened independently and any disagreements resolved by discussion or referral to a third reviewer. Methodological quality was assessed by Jadad Scale. ${ }^{11}$ For comparative studies we also assessed adequacy of sequence generation, allocation concealment and blinding.

Data relating to all aspects of the study was extracted by 1 reviewer using a standardized, piloted data extraction form in Access (Microsoft); a second reviewer checked all numerical data. Data was extracted on study and population characteristics, intervention and comparator characteristics, study quality and results.

\section{Statistical Analysis}

All trial data was combined using RevMan 5.2 (Cochrane Collaboration). Studies consisted of parallel RCTs, randomized crossover trials and randomized single assessment studies. Analysis of studies was dichotomized to those which involved structured pulmonary rehabilitation (PR) and those which were domiciliary. Only the results from the same exercise test protocols were combined. Pre- and post-test exercise outcomes reported for both intervention groups were from tests performed on compressed air.

Data was pooled with the generic inverse variance method using change from baseline with the appropriate means and standard deviations for each study if provided. For studies that reported pre- and posttraining means and respective standard deviations, the change in outcome measure pre-training means were subtracted from the post-training means and standard deviations (SD) for pre- and post-training were pooled.

A fixed effect was used in all analyses unless heterogeneity (defined as $\mathrm{I}^{2}>30 \%$ ) was present in which case a random effect was used. The meta-analyses are presented using forest plots from RevMan. Data not in a format suitable for meta-analysis have been reported in narrative form.

\section{Results}

\section{Description of Studies}

Twenty-three studies (620 patients) were included ${ }^{12-34}$ : 9 studies evaluated the clinical effectiveness of ambulatory oxygen and 14 evaluated the impact of the delivery device. Generally studies were small with primary outcomes focused on exercise capacity, Borg dyspnea scores and exercise saturations.

Figure 1 summarizes the trial flow in the PRISMA format for reporting systematic reviews. Tables 2 and 3 detail the characteristics of included studies. 


\section{Figure 1. Prisma Flow Diagram}
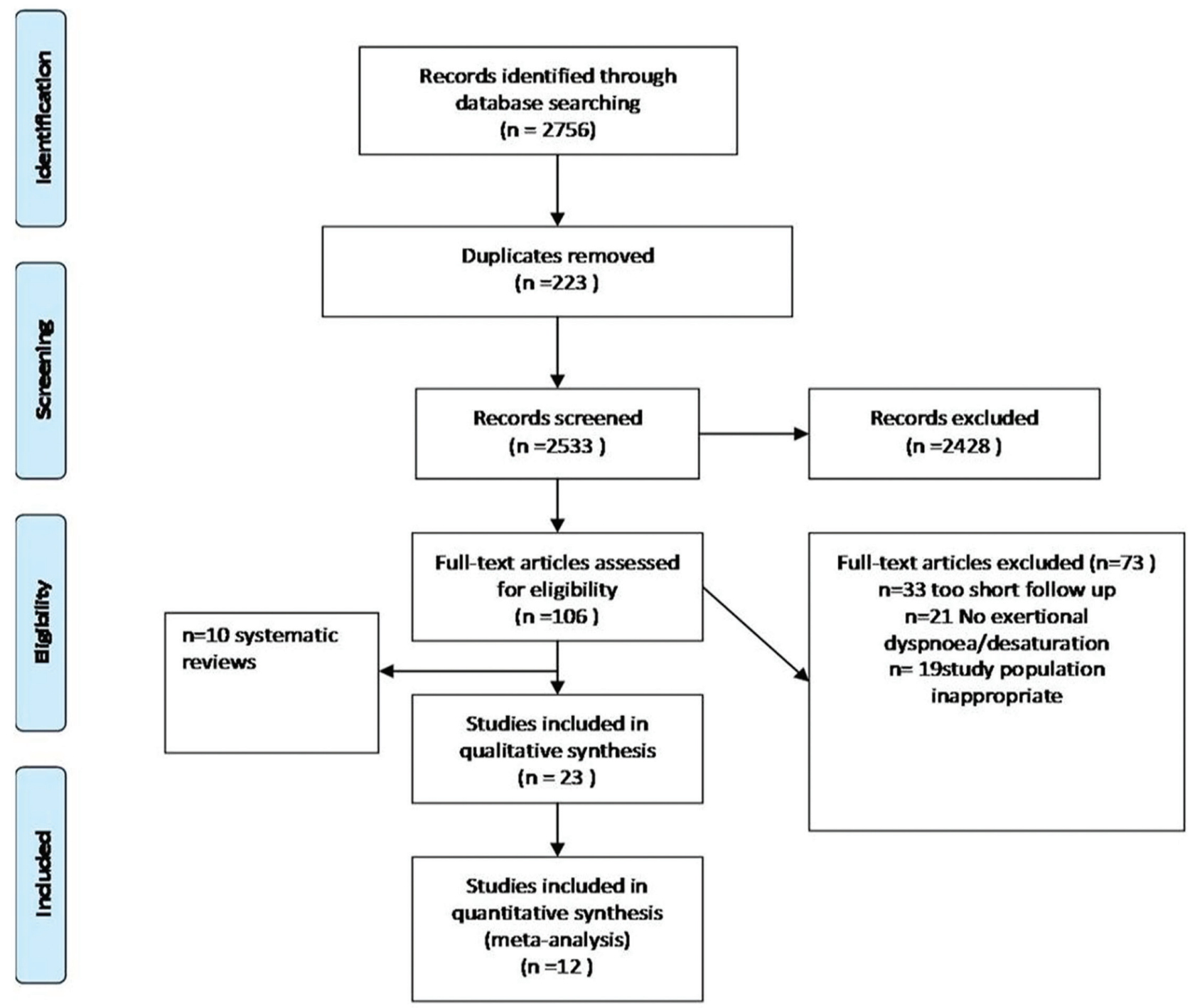

\section{Methodological Quality of Included Studies}

The quality of studies assessed by the Jadad scale was poor in 12 studies (score<2), good in 9 (score 3 or 4) and excellent in 2 (score 5) (Supplementary Table 1).

\section{Clinical Effectiveness of Ambulatory Oxygen}

\section{Exercise Capacity}

Four RCTs ${ }^{12,13,17,18}$ used 6MWT distance in assessing exercise capacity. In the PR studies ${ }^{13,17}$ the common effect (weighted mean difference) was 21.97 meters in favor of the control group. This result was mirrored in the meta-analysis of the domiciliary studies ${ }^{12,18}$; common effect 0.56 meters in favor of control. However, neither result was statistically significant (95\% confidence interval [CI] -77.25 to 33.32 ) and (95\% CI -26.96 to
28.08) respectively, Figure 2. Two PR RCTs ${ }^{19,20}$ used ESWT; they differed substantially in their results, hence the pooled figure may be less meaningful. It was not statistically significant with a common effect of 150.02 seconds in favor of oxygen (95\% CI -124.74 to 427.77 seconds, Figure 2). Two PR RCTs ${ }^{13,15}$ used incremental work rate exercise testing via cycle ergometry to assess exercise capacity and found no difference with oxygen; 1.45 watts in favor of control (95\% CI - 13.54 to 10.64 watts), Figure 2. Two PR RCTs ${ }^{13,15}$ measured constant power exercise tests using cycle ergometry. There was a strong trend toward longer exercise time with oxygen (2.76 minutes longer [95\% CI -0.07 to 5.58 minutes] Figure 2). 
Table 2. Clinical Effectiveness of Ambulatory Oxygen Studies

\begin{tabular}{|c|c|c|c|c|c|c|c|c|c|c|}
\hline Author & Year & Inclusion & Exclusion & $\begin{array}{l}\text { Study } \\
\text { Type }\end{array}$ & $\begin{array}{l}\text { Inter- } \\
\text { vention }\end{array}$ & $\begin{array}{l}\text { Comp- } \\
\text { arator }\end{array}$ & $\begin{array}{l}\text { Sample } \\
\text { Size }\end{array}$ & Methods & Outcomes & $\begin{array}{l}\text { Results } \\
\text { Summary }\end{array}$ \\
\hline $\begin{array}{l}\text { McDonald } \\
\text { et al } 12\end{array}$ & 1995 & $\begin{array}{l}\text { a) } \mathrm{COPD} \\
\text { b) } \mathrm{PaO}_{2}> \\
60 \mathrm{mmHg} / \\
8 \mathrm{kPa} \\
\text { c) Clinically } \\
\text { stable } \\
\text { d) Exertional } \\
\text { dyspnea } \\
\text { sufficient to } \\
\text { interfere with } \\
\text { ADLs } \\
\text { e) Current } \\
\text { non smokers }\end{array}$ & $\begin{array}{l}\text { a) Symptomatic } \\
\text { cardiac } \\
\text { dysfunction, } \\
\text { angina pectoris } \\
\text { and locomotor } \\
\text { disability }\end{array}$ & $\begin{array}{l}\text { Crossover } \\
\text { double- } \\
\text { blinded }\end{array}$ & $\begin{array}{l}\text { Oxygen } \\
41 / \mathrm{min}\end{array}$ & $\begin{array}{l}\text { Air } \\
41 / \min \end{array}$ & 26 & $\begin{array}{l}\text { Unsupervised } \\
12 \text { week } \\
\text { domiciliary } \\
\text { oxygen/air } \\
\text { use }\end{array}$ & $\begin{array}{l}\text { a) } 6 \mathrm{MWT} \\
\text { b) Step } \\
\text { test } \\
\text { c) Borg } \\
\text { Scores } \\
\text { d) CRQ }\end{array}$ & $\begin{array}{l}\text { Small significant } \\
\text { improvement } \\
\text { in 6MWT on home } \\
\text { oxygen; } \\
\text { Improvement in all } \\
\text { parameters } \\
\text { of CRQ on home } \\
\text { oxygen } \\
\text { compared to baseline. } \\
p<0.02 \text {. }\end{array}$ \\
\hline $\begin{array}{l}\text { Rooyackers } \\
\text { et al } 13\end{array}$ & 1997 & $\begin{array}{l}\text { a) } \mathrm{COPD} \\
\text { b) } \mathrm{SpO}_{2}< \\
90 \% \text { at } \\
\text { maximal } \\
\text { exercise } \\
\text { c) }>15 \mathrm{mmHg} / \\
2.00 \mathrm{kPa} \\
\text { increase in } \\
\text { alveolar-arterial } \\
\text { difference in } \\
\mathrm{PO}_{2} \text { from rest to } \\
\text { maximal exercise }\end{array}$ & $\begin{array}{l}\text { a) } \mathrm{PaO}_{2}<64 \mathrm{mmHg} / \\
8.53 \mathrm{kPa} \\
\text { b) Mean nocturnal } \\
\mathrm{SaO}_{2}<90 \% \\
\text { c) Mean pulmonary } \\
\text { artery pressure } \\
>25 \mathrm{mmHg} \\
\text { d) Neuromuscular } \\
\text { or cardiovascular } \\
\text { disease }\end{array}$ & $\begin{array}{l}\text { Parallel } \\
\text { un-blinded }\end{array}$ & $\begin{array}{l}\text { Oxygen } \\
41 / \mathrm{min}\end{array}$ & Air & $\begin{array}{l}12 \text { air } \\
\text { group, } \\
12 \\
\text { oxygen } \\
\text { group }\end{array}$ & $\begin{array}{l}10 \text { week } \\
\text { supervised PR } \\
\text { program; } 5 \\
\text { sessions/week }\end{array}$ & $\begin{array}{l}\text { a) PFTs } \\
\text { b) Maximal } \\
\text { cycle } \\
\text { ergometry } \\
\text { (watts) } \\
\text { c) Endurance } \\
\text { cycling time } \\
\text { d) } 6 \mathrm{MWT} \\
\text { e) CRQ } \\
\text { f) Borg } \\
\text { Scores }\end{array}$ & $\begin{array}{l}\text { Improvement in peak } \\
\text { WR in air group only } \\
p<0.01 \text {. } \\
\text { Improvement in 6MWT } \\
\text { and total CRQ scores } \\
\text { compared to baseline in } \\
\text { both groups, } p<0.01 \text {. }\end{array}$ \\
\hline $\begin{array}{l}\text { Eaton } \\
\text { et al }{ }^{14}\end{array}$ & 2002 & $\begin{array}{l}\text { a) } \mathrm{COPD} \\
\text { b) }<88 \% \mathrm{SpO}_{2} \text { on } \\
\text { exertion } \\
\text { c) Resting } \mathrm{PaO}_{2} \\
>7.3 \mathrm{kPa} \\
\text { d) clinically stable }\end{array}$ & $\begin{array}{l}\text { a) Limiting angina } \\
\text { or significant } \\
\text { musculoskeletal } \\
\text { disability }\end{array}$ & $\begin{array}{l}\text { Crossover } \\
\text { double- } \\
\text { blinded }\end{array}$ & $\begin{array}{l}\text { Oxygen } \\
41 / \mathrm{min}\end{array}$ & $\begin{array}{l}\text { Air } \\
41 / \min \end{array}$ & 41 & $\begin{array}{l}\text { Unsupervised } \\
12 \text { week } \\
\text { domiciliary } \\
\text { oxygen/air } \\
\text { use }\end{array}$ & $\begin{array}{l}\text { a) CRQ } \\
\text { b) HADs } \\
\text { c) SF-36 } \\
\text { d) } 6 \mathrm{MWT} \\
\text { e) Borg } \\
\text { Scores }\end{array}$ & $\begin{array}{l}\text { No significant difference } \\
\text { between groups in 6MWT, } \\
p=0.4 \text {. } \\
\text { Improvement in total CRQ } \\
\text { scores for home oxygen } \\
\text { period compared to home } \\
\text { air, } p=0.002 \text {. }\end{array}$ \\
\hline $\begin{array}{l}\text { Emtner } \\
\text { et al15 }\end{array}$ & 2003 & $\begin{array}{l}\text { a) } \mathrm{COPD} \text {; } \mathrm{FEV}_{1} \\
<50 \% \text { predicted } \\
\text { b) } 45-80 \text { years old } \\
\text { c) Clinically stable } \\
\text { d) } \mathrm{PaO}_{2}>55 \mathrm{mmHg} / \\
7.33 \mathrm{kPa} \text { at rest } \\
\text { e) } \mathrm{SpO}_{2}>88 \% \\
\text { during constant } \\
\text { WR test }\end{array}$ & $\begin{array}{l}\text { a) Symptomatic } \\
\text { cardiovascular } \\
\text { comorbidities or } \\
\text { other disease that } \\
\text { might contribute to } \\
\text { exercise limitation } \\
\text { b) Regular } \\
\text { participation in } \\
\text { formal exercise } \\
\text { program } \\
\text { c) Participation } \\
\text { within a formal } \\
\text { rehab program } \\
\text { in last } 2 \text { years }\end{array}$ & $\begin{array}{l}\text { Parallel } \\
\text { double- } \\
\text { blinded }\end{array}$ & $\begin{array}{l}\text { Oxygen } \\
31 / \mathrm{min}\end{array}$ & $\begin{array}{l}\text { Air } \\
\text { 31/min }\end{array}$ & $\begin{array}{l}14 \\
\text { oxygen } \\
\text { group, } \\
15 \text { air } \\
\text { group }\end{array}$ & $\begin{array}{l}\text { Supervised } 7 \\
\text { week exercise } \\
\text { program with } \\
3 \text { sessions/ } \\
\text { week }\end{array}$ & $\begin{array}{l}\text { a) Maximal } \\
\text { cycle } \\
\text { ergometry } \\
\text { (watts) } \\
\text { b) Constant } \\
\text { power cycle } \\
\text { ergometry } \\
\text { c) CRQ } \\
\text { d) SF-36 } \\
\text { e) Borg } \\
\text { Scores }\end{array}$ & $\begin{array}{l}\text { Improvement in peak } \\
\text { exercise tolerance test in } \\
\text { both groups, } p<0.05 \text {. } \\
\text { Endurance in constant } \\
\text { WR improved significantly } \\
\text { in oxygen group compared } \\
\text { to air group, } p<0.05 \text {. }\end{array}$ \\
\hline $\begin{array}{l}\text { Nonoyama } \\
\text { et al } 16\end{array}$ & 2007 & $\begin{array}{l}\text { a) COPD } \\
\text { b) }<88 \% \mathrm{SpO}_{2} \text { on } \\
6 \mathrm{MWT} \\
\text { c) Dyspnea } \\
\text { interfering with } \\
\text { ADLs }\end{array}$ & a) LTOT & $\begin{array}{l}\mathrm{N} \text { of } 1 \\
\mathrm{RCTs}\end{array}$ & $\begin{array}{l}\text { Oxygen } \\
21 / \mathrm{min}\end{array}$ & $\begin{array}{l}\text { Air } \\
21 / \min \end{array}$ & 27 & $\begin{array}{l}\text { Unsupervised } \\
6 \text { weeks } \\
\text { domiciliary } \\
\text { oxygen/air } \\
\text { use }\end{array}$ & $\begin{array}{l}\text { a) CRQ } \\
\text { b) SGRQ } \\
\text { c) } 5 M W T \\
\text { d) Borg } \\
\text { Scores }\end{array}$ & $\begin{array}{l}\text { Improvement in 5MWT } \\
\text { and Borg dyspnea scores } \\
\text { for home oxygen vs. home, } \\
\text { air } p=0.04 \text {. } \\
\text { No significant difference in } \\
\text { HRQoL between home } \\
\text { oxygen or home air. }\end{array}$ \\
\hline
\end{tabular}




\begin{tabular}{|c|c|c|c|c|c|c|c|c|c|}
\hline $\begin{array}{l}\text { Janaudis- } \\
\text { Ferreira } \\
\text { et al } 17\end{array}$ & $\begin{array}{l}2009 \text { a) } \mathrm{COPD} \mathrm{FEV}_{1}, 70 \% \\
\text { b) }<92 \% \mathrm{SpO}_{2} \text { on } \\
6 \mathrm{WMT} \\
\text { c) } \mathrm{PaO}_{2}>8 \mathrm{kPa} \text { at } \\
\text { rest } \\
\text { d) Clinically stable } \\
\text { e) Not smoking } \\
6 \text { months prior to } \\
\text { study }\end{array}$ & $\begin{array}{l}\text { a) Severe cardiac, } \\
\text { orthopaedic or } \\
\text { neurological } \\
\text { problems that } \\
\text { would influence } \\
\text { exercise } \\
\text { performance } \\
\text { b) LTOT }\end{array}$ & $\begin{array}{l}\text { Parallel } \\
\text { single- } \\
\text { blinded }\end{array}$ & $\begin{array}{l}\text { Oxygen } \\
51 / \mathrm{min}\end{array}$ & $\begin{array}{l}\text { Air } \\
51 / \mathrm{min}\end{array}$ & $\begin{array}{l}10 \text { air } \\
\text { group, } \\
10 \\
\text { oxygen } \\
\text { group }\end{array}$ & $\begin{array}{l}\text { Supervised } 8 \\
\text { week training } \\
\text { program }\end{array}$ & $\begin{array}{l}\text { a) } 6 \mathrm{MWT} \\
\text { b) Time } \\
<90 \% \\
\text { saturations } \\
\text { c) Borg Score }\end{array}$ & $\begin{array}{l}\text { Improvement in } 6 \mathrm{WMT} \text { in } \\
\text { both groups compared to } \\
\text { baseline } p<0.008 \text { air group, } \\
\text { and } p<0.005 \text { oxygen group. } \\
\text { Greater proportion of } \\
\text { patients achieve MCID for } \\
6 \mathrm{MWT} \text { in air group, } p=0.01 \text {. }\end{array}$ \\
\hline $\begin{array}{l}\text { Ringbaek } \\
\text { et al20 }\end{array}$ & $\begin{array}{l}2013 \text { a) } \mathrm{COPD} \text {; } \mathrm{FEV}_{1}<80 \% \\
\text { predicted } \\
\text { b) MRC } 3-5 \\
\text { c) Desaturation }>4 \% \\
\text { and below } 90 \% \text { on } \\
\text { ISWT } / E S W T \\
\text { d) } \mathrm{SpO}_{2}>90 \% \text { at rest }\end{array}$ & $\begin{array}{l}\text { a) LTOT } \\
\text { b) Significant } \\
\text { musculoskeletal, } \\
\text { cardiac or } \\
\text { cognitive } \\
\text { problems }\end{array}$ & $\begin{array}{l}\text { Parallel } \\
\text { un-blindec }\end{array}$ & $\begin{array}{l}\text { Oxygen } \\
\text { d21/min }\end{array}$ & & $\begin{array}{l}22 \\
\text { oxygen } \\
\text { group, } \\
23 \text { air } \\
\text { group }\end{array}$ & $\begin{array}{l}20 \text { week } \\
\text { training } \\
\text { program } \\
\text { supervised for } \\
\text { the first } 7 \\
\text { weeks }\end{array}$ & $\begin{array}{l}\text { a) ESWT } \\
\text { b) SGRQ }\end{array}$ & $\begin{array}{l}\text { Improvement in ESWT } \\
\text { (time and distance) in } \\
\text { both groups, } p<0.001\end{array}$ \\
\hline
\end{tabular}

COPD: chronic obstructive pulmonary disease; $\mathrm{PaO}_{2}$ : partial pressure of oxygen in arterial blood; ADLs: activities of daily living; $6 \mathrm{MWT}$ : 6 minute walk test; CRQ: Chronic Respiratory Questionnaire; $\mathrm{SaO}_{2}$ : oxygen saturation of arterial blood; $\mathrm{SpO}_{2}$ : blood oxygen saturation; HADS: Hamilton Depression and Anxiety Scale; PFTs: pulmonary function test, WR: work rate; 5MWT: 5 minute walk test; SGRQ: St. George's Respiratory Questionnaire;

HRQoL: Health-related Quality of Life; FEV1:forced expiratory volume in 1 second; BDI/TDI: baseline/transitional dyspnea scores; ISWT: incremental shuttle walk test; ESWT: endurance shuttle walk test;

Studies in bold denote structured pulmonary rehabilitation (PR) studies.

Two studies (both domiciliary) ${ }^{14,16}$ could not be used in the meta-analysis as 1 study provided $p$ values only ${ }^{14}$ and the other was the only one to use $5 \mathrm{MWT} .^{16}$ Neither showed any statistical benefit of long term ambulatory oxygen on $6 \mathrm{MWT}$ distance $(p=0.9)$ or $5 \mathrm{MWT}$ distance for individual participants.

\section{Borg Dyspnea Scores}

End of test Borg scores were measured in 2 of the meta-analysed PR studies that used $6 \mathrm{MWT}^{13,17}$ and 2 RCTs using incremental work exercise testing. ${ }^{13,15}$ Heterogeneity between the studies was not significant $\left(\mathrm{I}^{2}=0 \%\right)$. Neither were statistically significant; effect 0.16 $(-0.61$ to 0.93$)$ and 0.25 (-0.81 to 1.31 ) respectively; (Figure 3).

\section{Quality of Life}

This was measured by the Chronic Respiratory Disease
Questionnaire (CRQ) in 7 studies $^{12-16,18,19} 3$ of which were PR studies. In the PR studies there was no statistical difference between oxygen and control in any domain, however there was a strong trend to statistical significance in $\mathrm{CRQ}$ emotion and fatigue favoring oxygen; $p=0.06$ and $p=0.09$ respectively (Figure 4 ). In the domiciliary studies the effect in all domains was in favor of oxygen treatment, significant only in dyspnea $(p=0.002)$ and fatigue $(p=0.01)$. (Figure 5 )

\section{Impact of Device on Ambulatory Oxygen Delivery}

Fourteen studies evaluated a variety of oxygen conserving devices (OCDs) and cylinder types ${ }^{21-34}$ (Table 3). Modes of delivery included demand oxygen devices (DODs), pendant reserve cannulae (PRC) and continuous flow nasal cannulae (CFNC). In some studies, multiple $O C D$ s were compared. Where this was the case, the most effective OCD was used in the meta- 


\begin{tabular}{|c|c|c|c|c|c|c|c|c|c|c|}
\hline Author & Year & Inclusion & Exclusion & $\begin{array}{l}\text { Study } \\
\text { Type }\end{array}$ & $\begin{array}{l}\text { Inter- } \\
\text { vention }\end{array}$ & $\begin{array}{l}\text { Comp- } \\
\text { arator }\end{array}$ & $\begin{array}{l}\text { Sample } \\
\text { Size }\end{array}$ & Methods & Outcomes & $\begin{array}{l}\text { Results } \\
\text { Summary }\end{array}$ \\
\hline $\begin{array}{l}\text { Soffer } \\
\text { et } \mathrm{al}^{21}\end{array}$ & 1985 & $\begin{array}{l}\mathrm{COPD} \text { or restrictive } \\
\text { ventilatory disease. } \\
\mathrm{SpO}_{2}<90 \%\end{array}$ & NR & $\begin{array}{l}\text { Non-RCT } \\
\text { crossover }\end{array}$ & PRC & CFNC & $\begin{array}{l}20 \\
(13 \\
\text { COPD) }\end{array}$ & $\begin{array}{l}\text { Treadmill } \\
\text { exercise }\end{array}$ & $\begin{array}{l}\text { a) Oxygen } \\
\text { conservation } \\
\text { ratio at } 90 \% \\
\mathrm{SpO}_{2} \\
\text { b) } \% \text { oxygen } \\
\text { savings }\end{array}$ & $\begin{array}{l}\text { PRC requires lower flow } \\
\text { rate to achieve } 90 \% \\
\text { saturations compared to } \\
\text { CFNC. } p<0.05 \text { (data from } \\
\text { COPD patients alone) }\end{array}$ \\
\hline $\begin{array}{l}\text { Carter } \\
\text { et } \mathrm{al}^{22}\end{array}$ & 1986 & $\begin{array}{l}\text { COPD exercise } \\
\text { desaturation } \\
\text { Resting } \mathrm{SpO}_{2} \\
\leq 90 \%\end{array}$ & NR & Crossover & PRC & CFNC & 10 & $\begin{array}{l}\text { Treadmill } \\
\text { exercise test } \\
(1-1.5 \mathrm{mph})\end{array}$ & $\begin{array}{l}\mathrm{SpO}_{2} \text { on } \\
\text { exercise }\end{array}$ & $\begin{array}{l}\mathrm{PRC} \text { achieves statistically } \\
\text { higher } \mathrm{SpO}_{2} \text { on exercise } \\
\text { compared to } \mathrm{CFNC} \text {, } \\
p<0.001 .\end{array}$ \\
\hline $\begin{array}{l}\text { Tiep } \\
\text { et } \mathrm{al}^{23}\end{array}$ & 1987 & COPD & $\begin{array}{l}\text { No cardiovascular } \\
\text { or orthopaedic } \\
\text { limitations to } \\
\text { exercise }\end{array}$ & $\begin{array}{l}\text { Non-RCT } \\
\text { crossover }\end{array}$ & DODs & CFNC & 9 & $\begin{array}{l}\text { Treadmill } \\
\text { exercise test }\end{array}$ & $\begin{array}{l}\text { Oxygen flow } \\
\text { setting } \\
\text { required to } \\
\text { achieve } \mathrm{SpO}_{2} \\
>90 \%\end{array}$ & $\begin{array}{l}\text { DODs requires lower flow } \\
\text { rate to achieve } 90 \% \\
\text { saturations, } p<0.0001 \text {. }\end{array}$ \\
\hline $\begin{array}{l}\text { Bower } \\
\text { et } \mathrm{al}^{24}\end{array}$ & 1988 & $\begin{array}{l}\text { Chronic lung } \\
\text { disease; } \\
\text { Clinically stable }\end{array}$ & NR & Crossover & DODs & CFNC & $\begin{array}{l}5 \\
\text { COPD } \\
1 \\
\text { COPD/ } \\
\text { scoliosis }\end{array}$ & $\begin{array}{l}\text { Treadmill } \\
\text { exercise test }\end{array}$ & $\begin{array}{l}\text { a) Oxygen } \\
\text { usage } \\
\text { percentage } \\
\text { b) Exercise } \\
\mathrm{SpO}_{2}\end{array}$ & $\begin{array}{l}\text { No significant difference in } \\
\text { exercise } \mathrm{SpO}_{2} \text { between } \\
\text { DODs and CFNC. } \\
\text { DODs mean oxygen } \\
\text { utilization is } 44 \% \text { that of } \\
\text { CFNC oxygen utilization. }\end{array}$ \\
\hline $\begin{array}{l}\text { Braun } \\
\text { et al } 25\end{array}$ & 1992 & $\begin{array}{l}\text { COPD: } \mathrm{FEV}_{1} / \\
\text { FVC ratio }<60 \% \text {, } \\
\text { LTOT }\end{array}$ & NR & Crossover & $\begin{array}{l}\text { DODs } \\
\text { (5 } \\
\text { devices) }\end{array}$ & CFNC & 10 & $\begin{array}{l}12 \text { minute } \\
\text { walk test }\end{array}$ & $\begin{array}{l}\text { Average and } \\
\text { lowest } \mathrm{SpO}_{2} \\
\text { at rest and } \\
\text { exertion }\end{array}$ & $\begin{array}{l}\text { No significant difference } \\
\text { between the mean of the } \\
\text { lowest } \mathrm{SpO}_{2} \text { during } \\
\text { exercise between } \\
\text { intervention and } \\
\text { comparator. }\end{array}$ \\
\hline $\begin{array}{l}\text { Roberts } \\
\text { et } \mathrm{al}^{26}\end{array}$ & 1995 & $\begin{array}{l}\text { COPD } \\
\text { exercise } \\
\text { desaturation } \\
<90 \% \text {; } \\
\text { Clinically stable }\end{array}$ & NR & Crossover & DODs & CFNC & 15 & $6 \mathrm{MWT}$ & $\begin{array}{l}\text { a) } 6 \mathrm{MWT} \\
\text { b) time spent } \\
<90 \% \mathrm{SpO}_{2}\end{array}$ & $\begin{array}{l}\text { CFNC significantly } \\
\text { improved } 6 \mathrm{MWD} \text { and } \\
\text { reduced time }<90 \% \mathrm{SpO}_{2} \\
\text { (compared to baseline) } \\
\text { whereas DODs only } \\
\text { improved 6MWT. }\end{array}$ \\
\hline $\begin{array}{l}\text { Cuvelier } \\
\text { et } \mathrm{al}^{27}\end{array}$ & 2002 & $\begin{array}{l}\text { COPD-ATS criteria; } \\
\text { clinically stable } \\
\mathrm{FEV}_{1} / \mathrm{FVC}<65 \% \\
\mathrm{FEV}_{1}<55 \% \\
\mathrm{PaO}_{2} \leq 60 \mathrm{mmHg} \text { at } \\
\text { rest } / \mathrm{PaO}_{2} \\
>60 \mathrm{mmHg} \text { with } \\
\text { evidence of exercise } \\
\text { desaturation }\end{array}$ & NR & Crossover & 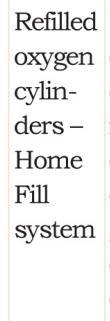 & $\begin{array}{l}\text { Stan- } \\
\text { dard } \\
\text { com- } \\
\text { mer- } \\
\text { cial } \\
\text { oxy- } \\
\text { gen } \\
\text { cylin- } \\
\text { ders }\end{array}$ & 10 & $\begin{array}{l}\text { Successive } \\
6 \mathrm{MWT} \\
\text { carrying } \\
\text { standard } \\
\text { oxygen and } \\
\text { refilled oxygen } \\
\text { cylinders }\end{array}$ & $\begin{array}{l}\text { a) } 6 \mathrm{MWD} \\
\text { b) Mean } \\
\mathrm{SpO}_{2} \text { at end } \\
\text { of } 6 \mathrm{MWT} \\
\end{array}$ & $\begin{array}{l}\text { No significant difference } \\
\text { between } 6 \mathrm{MWD} \text { or } \mathrm{SpO}_{2} \\
\text { between cylinder types. }\end{array}$ \\
\hline $\begin{array}{l}\text { Tiep } \\
\text { et } \mathrm{al}^{28}\end{array}$ & 2002 & $\begin{array}{l}\text { Chronic lung } \\
\text { disease; } \\
\text { Desaturation on } \\
\text { exercise; } \\
\text { Clinically stable; } \\
\text { All patients using } \\
\text { supplementary } \\
\text { oxygen }\end{array}$ & NR & $\begin{array}{l}\text { Non-RCT } \\
\text { crossover }\end{array}$ & DODs & CFNC & $\begin{array}{l}10 \\
(9 \\
\text { COPD) }\end{array}$ & $\begin{array}{l}\text { Treadmill } \\
\text { exercise test; } \\
\text { oxygen flow } \\
\text { required to } \\
\text { maintain } \\
\text { saturations } \\
\text { between } 92 \\
\text { and } 94 \%\end{array}$ & $\begin{array}{l}\text { Oxygen flow } \\
\text { setting } \\
\text { required to } \\
\text { achieve } \mathrm{SpO}_{2} \\
>90 \%\end{array}$ & $\begin{array}{l}\text { No significant difference in } \\
\mathrm{SpO}_{2} \text { at higher DODs } \\
\text { setting. DODs confer } 4.3 \\
\text { fold oxygen saving on } \\
\text { exercise. }\end{array}$ \\
\hline
\end{tabular}




\begin{tabular}{|c|c|c|c|c|c|c|c|c|c|c|}
\hline $\begin{array}{l}\text { Chatila } \\
\text { et al } 29\end{array}$ & 2004 & $\begin{array}{l}4 \text { COPD-GOLD } \\
\text { criteria; } \\
\text { Clinically stable the } \\
\text { preceding } 3 \text { months }\end{array}$ & $\begin{array}{l}\text { Cardiovascular } \\
\text { disease }\end{array}$ & $\begin{array}{l}\text { Non-RCT } \\
\text { crossover }\end{array}$ & $\begin{array}{l}\text { Vapo- } \\
\text { therm } \\
\text { (high } \\
\text { flow } \\
\text { oxygen } \\
\text { 201/ } \\
\text { min) }\end{array}$ & $\begin{array}{l}\text { Low } \\
\text { flow } \\
\text { oxy- } \\
\text { gen } \\
(2.5- \\
61 / \\
\text { min) }\end{array}$ & $\begin{array}{l}10 \\
(5 \\
\text { com- } \\
\text { pleted } \\
\text { exer- } \\
\text { cise } \\
\text { test) }\end{array}$ & $\begin{array}{l}\text { Cycling- } \\
\text { unloaded } \\
\text { bicycle }\end{array}$ & $\begin{array}{l}\text { a) } V_{T} \\
\text { b) } V_{E} \\
\text { c) Work of } \\
\text { breathing } \\
\text { d) Inspiratory } \\
\text { time fraction } \\
\text { e) RR/VT }\end{array}$ & $\begin{array}{l}\text { No significant difference in } \\
\text { outcome measure } \\
\text { (a,b or c) between } \\
\text { vapotherm or low } \\
\text { flow oxygen. }\end{array}$ \\
\hline $\begin{array}{l}\text { Fuhrman } \\
\text { et al30 }\end{array}$ & 2004 & $\begin{array}{l}4 \mathrm{COPD}_{\mathrm{FEV}}<50 \% \text {; } \\
\mathrm{PaO}_{2}<65 \mathrm{mmHg} \text {; } \\
\text { Current or former } \\
\text { smoker; } \\
\text { No exacerbations } \\
\text { in preceding } 3 \\
\text { months }\end{array}$ & $\begin{array}{l}\text { Symptomatic } \\
\text { cardiovascular } \\
\text { disease; Any } \\
\text { condition with } \\
\text { contraindication } \\
\text { to exercise testing }\end{array}$ & Crossover & $\begin{array}{l}\text { DODs } \\
\text { ( } 4 \mathrm{dev}- \\
\text { ices) }\end{array}$ & CFNC & 13 & $6 \mathrm{MWT}$ & $\begin{array}{l}\text { a) } \mathrm{SpO}_{2} \text { at } \\
\text { rest and } \\
\text { exercise } \\
\text { b) } 6 \mathrm{MWT}\end{array}$ & $\begin{array}{l}\text { No significant difference in } \\
\text { 6MWD with any DODs } \\
\text { device compared with } \\
\text { CFNC. }\end{array}$ \\
\hline $\begin{array}{l}\text { Strickland } \\
\text { et } \mathrm{al}^{31}\end{array}$ & 2009 & $\begin{array}{l}9 \text { COPD-GOLD Stage } \\
\text { 4; } \mathrm{SpO}_{2}<90 \% ; \\
\text { LTOT; } \\
\text { Clinically stable } \\
\text { preceding } 6 \text { weeks }\end{array}$ & $\begin{array}{l}\text { Symptomatic } \\
\text { cardiovascular } \\
\text { disease; Physical } \\
\text { limitations } \\
\text { precluding walking }\end{array}$ & Crossover & $\begin{array}{l}\text { a)Liquic } \\
\text { oxygen } \\
\text { system } \\
\text { b)Home } \\
\text { Fill } \\
\text { comp- } \\
\text { ressed } \\
\text { oxygen } \\
\text { system } \\
\text { c)Port- } \\
\text { able } \\
\text { oxygen } \\
\text { concen- } \\
\text { trator }\end{array}$ & $\begin{array}{l}\text { d Stan- } \\
\text { dard } \\
\text { com- } \\
\text { e mer- } \\
\text { cial } \\
\text { oxy- } \\
\text { gen } \\
\text { cylin- } \\
\text { ders }\end{array}$ & 39 & $6 \mathrm{MWT}$ & $\begin{array}{l}\mathrm{SpO}_{2} \text { on } \\
\text { exercise }\end{array}$ & $\begin{array}{l}\text { No significant difference } \\
\text { between } \mathrm{SpO}_{2} \text { between } \\
\text { interventions or } \\
\text { comparator. }\end{array}$ \\
\hline $\begin{array}{l}\text { Palwai } \\
\text { et } \mathrm{al}^{32}\end{array}$ & 2010 & $\begin{array}{l}\text { Obstructive lung } \\
\text { disease; } \mathrm{SpO}_{2} \\
<90 \% \text { at rest or on } \\
\text { exertion; } \\
\text { Hypoxaemia } \\
\text { warranting use of } \\
\text { supplementary } \\
\text { oxygen }\end{array}$ & NR & Crossover & $\begin{array}{l}\text { DODs } \\
(4 \mathrm{dev}- \\
\text { ices })\end{array}$ & CFNC & 13 & $\begin{array}{l}\text { Treadmill } \\
\text { exercise test; } \\
\text { Modified } \\
\text { Naugton } \\
\text { protocol }\end{array}$ & $\begin{array}{l}\text { a) Work } \\
\text { stages } \\
\text { completed } \\
\text { b) } \mathrm{SpO}_{2} \\
\text { c) } \mathrm{VE}_{\mathrm{E}}\end{array}$ & $\begin{array}{l}\text { Significantly higher METs } \\
\text { achieved with CFNC, } \\
p=0.006 \text {. }\end{array}$ \\
\hline $\begin{array}{l}\text { Casaburi } \\
\text { et al } 33\end{array}$ & 2012 & $\begin{array}{l}\mathrm{COPD} \mathrm{FEV}_{1}<60 \% \text {; } \\
\mathrm{PaO}_{2}<8 \mathrm{kPa} \\
\text { Clinically stable } \\
\text { preceding month }\end{array}$ & $\begin{array}{l}\text { Uncontrolled heart } \\
\text { failure; Orthopedic, } \\
\text { neurological or } \\
\text { cognitive limitations } \\
\text { to exercise; } \\
\text { Current smokers }\end{array}$ & $\begin{array}{l}\text { RCT } \\
\text { parallel }\end{array}$ & $\begin{array}{l}\text { Light- } \\
\text { weight } \\
\text { alumi- } \\
\text { nium } \\
\text { cylin- } \\
\text { ders }\end{array}$ & $\begin{array}{l}\text { E } \\
\text { cylin- } \\
\text { ders }\end{array}$ & 22 & $\begin{array}{l}\text { Unsuper- } \\
\text { vised } \\
\text { domestic } \\
\text { activity }\end{array}$ & $\begin{array}{l}\text { Oxygen } \\
\text { utilization; } \\
\text { Home } \\
\text { activity }\end{array}$ & $\begin{array}{l}\text { No significant difference in } \\
\text { oxygen utilization or } \\
\text { patterns of activity } \\
\text { between lightweight and } \\
\text { standard cylinder. }\end{array}$ \\
\hline $\begin{array}{l}\text { Marti } \\
\text { et } \text { al }^{34}\end{array}$ & 2013 & $\begin{array}{l}\text { COPD and } \\
\text { interstitial lung } \\
\text { disease; Exercise } \\
\text { desaturation }<88 \%\end{array}$ & $\begin{array}{l}\text { Active smoker; } \\
\text { Use of ambulatory } \\
\text { oxygen therapy } \\
\text { prior to study; } \\
\text { Recent } \\
\text { exacerbation }\end{array}$ & Crossover & $\begin{array}{l}\text { DODs } \\
\text { PRC }\end{array}$ & CFNC & $\begin{array}{l}28 \\
\text { COPD } \\
31 \\
\text { inter- } \\
\text { stitial } \\
\text { lung } \\
\text { disease }\end{array}$ & $\begin{array}{l}\text { 6MWT using } \\
\text { intervention/ } \\
\text { comparator }\end{array}$ & $\begin{array}{l}\text { a) } \% \text { of } \\
\text { patients with } \\
\text { correction of } \\
\text { exercise } \\
\text { desaturation } \\
\text { b) } 6 \mathrm{MWD} \\
\text { c) Borg scores }\end{array}$ & $\begin{array}{l}\text { Exercise desaturation } \\
\text { corrected in } 79 \% \text { of } \\
\text { patients with DODs and } \\
\text { CFNC and in } 86 \% \text { with } \\
\text { PRC (data for COPD } \\
\text { patients alone). No } \\
\text { S significant difference } \\
\text { between devices. }\end{array}$ \\
\hline
\end{tabular}

COPD: chronic obstructive pulmonary disease; NR: not reported; CFNC: continuous flow nasal cannulae; RCT: randomized controlled trial; PRC: pendant reservoir cannulae; DODs: demand oxygen devices; FEV1: forced expiratory volume in 1 second; FVC: forced vital capacity; LTOT: long-term oxygen therapy; 6MWT: 6-minute walk test; ATS: American Thoracic Society; $\mathrm{PaO}_{2}$ : partial pressure of oxygen in arterial blood; GOLD: Global initiative for chronic Obstructive Pulmonary Disease; 6MWD: 6-minute walk distance; METS: metabolic equivalents

analysis comparing it to standard CFNC.

Five OCD studies ${ }^{22,24,26,30,34}$ were used in 2 separate meta-analyses. Tests for heterogeneity were not significant (both $\left.\mathrm{I}^{2}=0\right)$.

\section{Exercise Capacity}

Three of the 5 meta-analysed studies compared DODs against standard CFNC using 6MWT as an outcome measure. ${ }^{26,30,34}$ DODs and CFNC did not differ (CI- 


\section{Figure 2. Effect of Ambulatory Oxygen on Exercise Capacity}
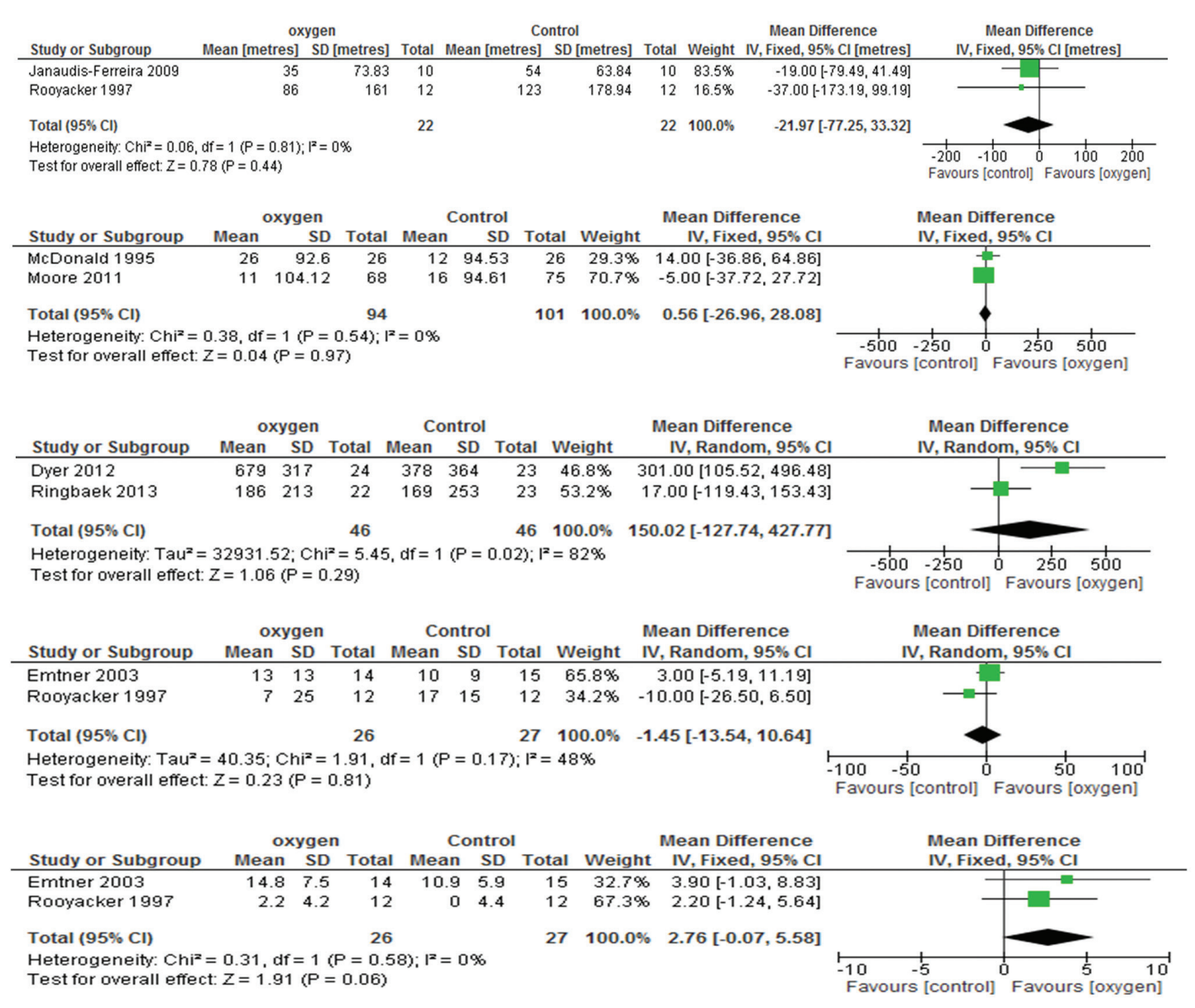

$2.16 \mathrm{MWT}$ (metres)

$2.26 \mathrm{MWT}$ (domiciliary studies)

\subsection{ESWT} (seconds)

2.4 Incremental power exercise (watts)

2.5 Constant work rate (mins)

All were rehab studies unless otherwise stated. No exercise outcomes showed significant benefit of ambulatory oxygen although there was a trend towards benefit when using constant work rate as the outcome measure $(p=0.06)$

\section{Figure 3. Effect of Ambulatory Oxygen on Exercise-induced Dyspnea}

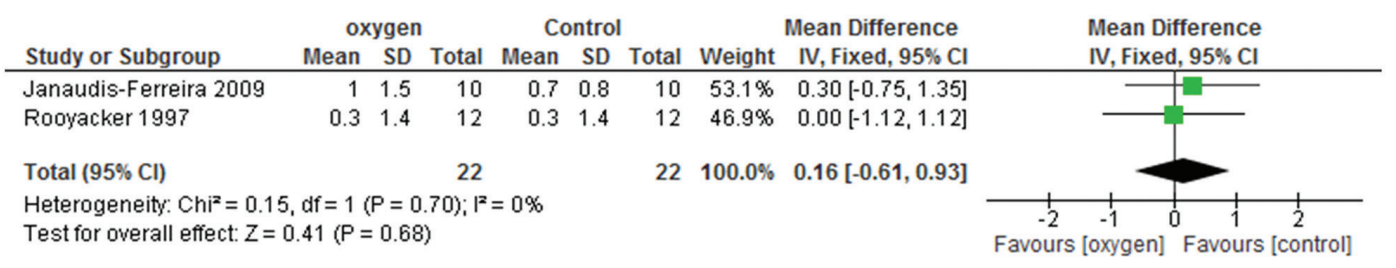

\subsection{Borg Score} 6MWT

\author{
3.2 Borg Score \\ incremental \\ power exercise
}




\section{Figure 4. Effect of Ambulatory Oxygen on HRQoL (Domiciliary Studies)}

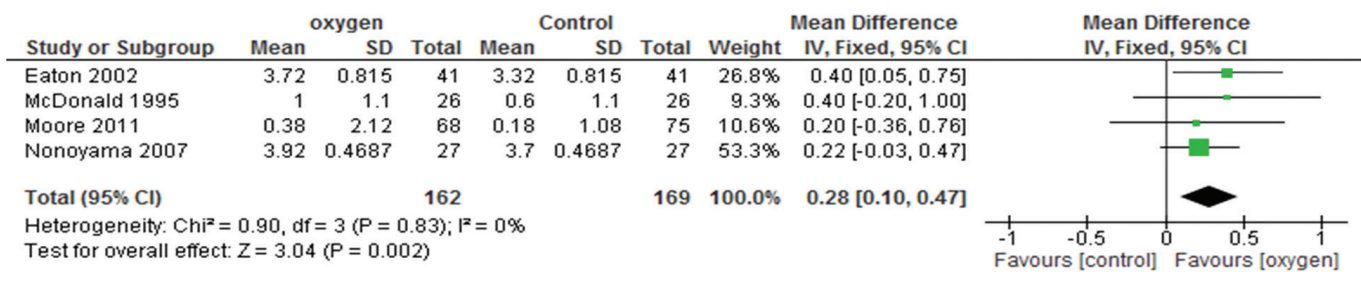

\subsection{CRQ Dyspnoea}

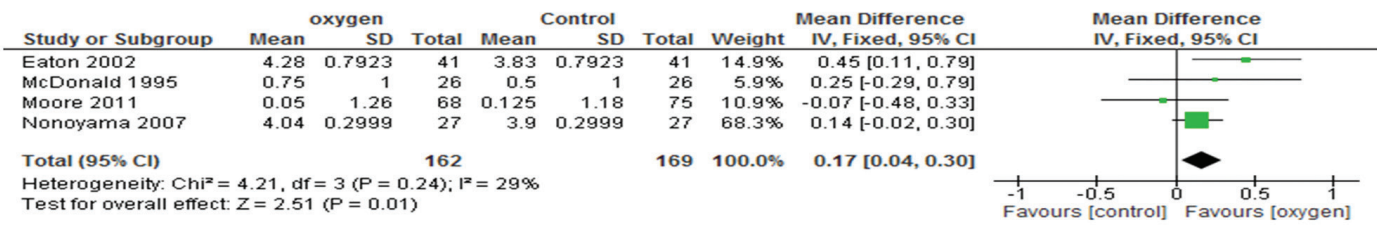

4.2 CRQ Fatigue

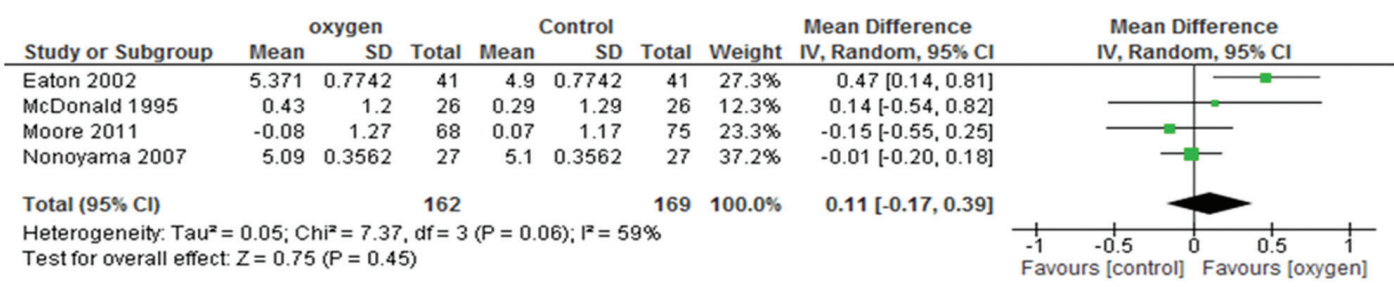

4.3 CRQ Emotion

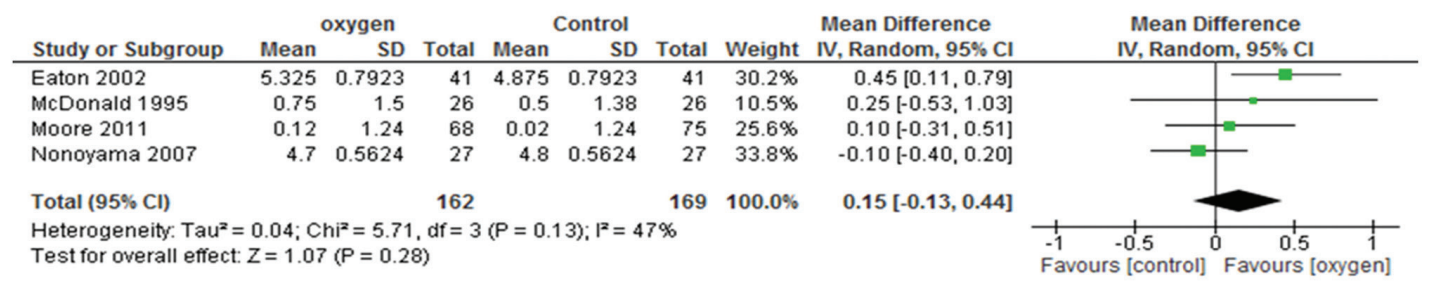

4.4 CRQ Mastery

10.28 to 1.60 ), (Figure 6).

\section{Exercise Oxygen Saturations}

Oxygen saturations were used in 2 of the 5 studies ${ }^{22,24}$ with methods of delivery being PRC and DODs respectively. Oxygen saturations were $2.03 \%$ higher with OCDs compared to CFNC, (95\% CI 0.09 to 3.98, $p=0.04$, Figure 6). In contrast, Roberts et al demonstrated that DODs caused a significantly longer period of desaturation $<90 \%$ than CFNC $p<0.001$.

\section{Oxygen Utilization}

Three studies ${ }^{21,23,28}$ evaluated the ability of OCDs versus standard CFNC to conserve oxygen in patients during exercise by calculating an oxygen conservation ratio at $90 \%$ blood oxygen saturation $\left(\mathrm{SpO}_{2}\right)$ (i.e., the relative difference in oxygen flow required to maintain oxygen saturation with both devices at $90 \% \mathrm{SpO}_{2}$ ). Two studies used DODs ${ }^{23,28}$ and $1 \mathrm{PRC}^{21}$ Although a higher DODs setting was required to maintain equivalent oxygen saturations, DODs still conferred 2.9-7.6 fold oxygen savings which was significant when compared to standard CFNC $p<0.001$. A similar result was demonstrated with PRC; oxygen utilization was significantly lower than CFNC $p<0.05 .^{21}$

\section{Refillable Cylinders and Home Activity}

Individual studies demonstrated no significant difference in exercise capacity with either refilled cylinders or portable oxygen concentrators when compared to standard commercial cylinders. ${ }^{27,31}$ Patterns of either home activity or oxygen utilization were not significantly different when lightweight cylinders were used compared to heavier E cylinders. ${ }^{33}$

\section{Cost Effectiveness}

There were no results from the searches assessing cost incremental ratios or cost effectiveness for ambulatory oxygen in addition to usual care. 


\section{Figure 5. Effect of Ambulatory Oxygen on HRQoL (PR Studies)}

\begin{tabular}{|c|c|c|c|c|c|c|c|c|c|c|}
\hline \multirow[b]{2}{*}{ Study or Subgroup } & \multicolumn{3}{|c|}{ oxygen } & \multicolumn{3}{|c|}{ Control } & \multicolumn{2}{|c|}{ Mean Difference } & \multirow{2}{*}{$\begin{array}{l}\text { Mean Difference } \\
\text { IV, Fixed, } 95 \% \mathrm{CI}\end{array}$} & \\
\hline & Mean & SD & Total & Mean & SD & Total & Weight & IV, Fixed, $95 \% \mathrm{Cl}$ & & \\
\hline Dyer 2012 & 0.4 & 1.3 & 24 & 0.6 & 1.3 & 23 & $45.1 \%$ & $-0.20[-0.94,0.54]$ & & \\
\hline Emtner 2003 & 1.6 & 1.2 & 14 & 1.4 & 1.8 & 15 & $20.3 \%$ & $0.20[-0.91,1.31]$ & & \\
\hline Rooyacker 1997 & 1.14 & 1.08 & 12 & 1.34 & 1.04 & 12 & $34.6 \%$ & $-0.20[-1.05,0.65]$ & & \\
\hline Total $(95 \% \mathrm{Cl})$ & & & 50 & & & 50 & $100.0 \%$ & $-0.12[-0.62,0.38]$ & & \\
\hline \multicolumn{9}{|c|}{$\begin{array}{l}\text { Heterogeneity: } \mathrm{Chi}^{2}=0.40, \mathrm{df}=2(P=0.82) ; \mathrm{I}^{2}=0 \% \\
\text { Test for overall effect: } Z=0.47(P=0.64)\end{array}$} & $\begin{array}{cccc}-2 & -1 & 0 & 1 \\
\text { Favours [control] } & \text { Favours }\end{array}$ & $\begin{array}{l}1 \\
2 \\
\text { oxygen] }\end{array}$ \\
\hline
\end{tabular}

5.1 CRQ Dyspnoea

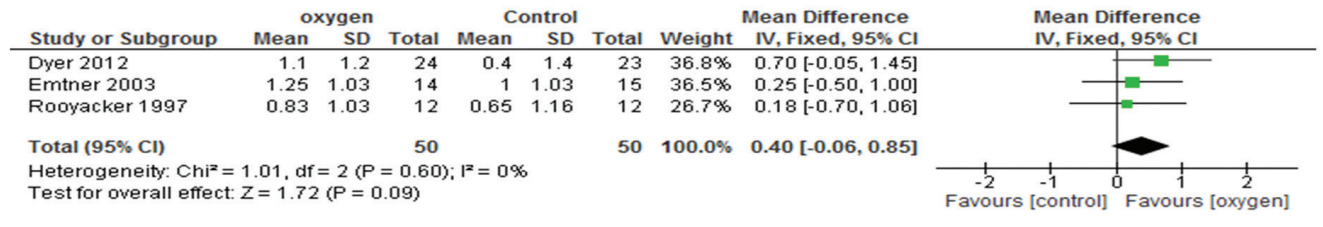

5.2 CRQ Fatigue

\begin{tabular}{|c|c|c|c|c|c|c|c|c|c|c|}
\hline \multirow[b]{2}{*}{ Study or Subgroup } & \multicolumn{3}{|c|}{ oxygen } & \multicolumn{3}{|c|}{ Control } & \multicolumn{2}{|c|}{ Mean Difference } & \multirow{2}{*}{$\begin{array}{l}\text { Mean Difference } \\
\text { IV, Fixed, } 95 \% \mathrm{Cl}\end{array}$} & \\
\hline & Mean & SD & Total & Mean & SD & Total & Weight & IV, Fixed, $95 \% \mathrm{Cl}$ & & \\
\hline Dyer 2012 & 0.7 & 1 & 24 & 0.2 & 1.2 & 23 & $43.5 \%$ & $0.50[-0.13,1.13]$ & & \\
\hline Emtner 2003 & 0.86 & 1.15 & 14 & 0.57 & 0.87 & 15 & $31.3 \%$ & $0.29[-0.46,1.04]$ & & \\
\hline Rooyacker 1997 & 0.83 & 0.96 & 12 & 0.46 & 1.11 & 12 & $25.3 \%$ & $0.37[-0.46,1.20]$ & & \\
\hline Total $(95 \% \mathrm{Cl})$ & & & 50 & & & 50 & $100.0 \%$ & $0.40[-0.02,0.82]$ & & \\
\hline $\begin{array}{l}\text { Heterogeneity: } \mathrm{Chi}^{2} \\
\text { Test for overall effect }\end{array}$ & $\begin{array}{l}0.18, d f \\
Z=1.89\end{array}$ & $\begin{array}{l}=2(P \\
(P=0\end{array}$ & $\begin{array}{l}=0.91) \\
.06)\end{array}$ & & & & & & $\begin{array}{cccc} & 1 & 1 & 1 \\
-2 & -1 & 0 & 1 \\
\text { Favours [control] } & \text { Favour }\end{array}$ & $\begin{array}{l}1 \\
2 \\
x y g e n]\end{array}$ \\
\hline
\end{tabular}

5.3 CRQ Emotion

\begin{tabular}{|c|c|c|c|c|c|c|c|c|c|c|}
\hline \multirow[b]{2}{*}{ Study or Subgroup } & \multicolumn{3}{|c|}{ oxygen } & \multicolumn{3}{|c|}{ Control } & \multirow{2}{*}{\multicolumn{2}{|c|}{$\begin{array}{c}\text { Mean Difference } \\
\text { Weight } \quad \text { IV, Fixed, } 95 \% \mathrm{Cl}\end{array}$}} & \multirow{2}{*}{\multicolumn{2}{|c|}{$\begin{array}{l}\text { Mean Difference } \\
\text { IV, Fixed, } 95 \% \mathrm{Cl}\end{array}$}} \\
\hline & Mean & SD & Total & Mean & SD & Total & & & & \\
\hline Dyer 2012 & 0.7 & 1.4 & 24 & 0.1 & 1 & 23 & $41.5 \%$ & $0.60[-0.09,1.29]$ & & -1 \\
\hline Emtner 2003 & 1 & 1.19 & 14 & 1 & 1 & 15 & $30.9 \%$ & $0.00[-0.80,0.80]$ & & \\
\hline Rooyacker 1997 & 1.03 & 1.14 & 12 & 0.6 & 0.98 & 12 & $27.6 \%$ & $0.43[-0.42,1.28]$ & & \\
\hline Total $(95 \% \mathrm{Cl})$ & & & 50 & & & 50 & $100.0 \%$ & $0.37[-0.08,0.81]$ & & \\
\hline $\begin{array}{l}\text { Heterogeneity: } \mathrm{Chi}^{2}= \\
\text { Test for overall effect }\end{array}$ & $\begin{array}{l}1.26, \mathrm{df} \\
Z=1.61\end{array}$ & $\begin{array}{l}=2(P \\
(P=0\end{array}$ & $\begin{array}{l}=0.53) \\
.11)\end{array}$ & $I^{2}=0 \%$ & & & & & $\begin{array}{cc}1 & -1 \\
\text { Favours [control] }\end{array}$ & $\begin{array}{ccc} & 1 & 1 \\
0 & 1 & 2 \\
\text { Favours [oxygen] }\end{array}$ \\
\hline
\end{tabular}

5.4 CRQ Mastery

\section{Discussion}

This review evaluated the use of ambulatory oxygen in 3 separate ways: efficacy against placebo, optimum mode of delivery and cost. First, we have shown that ambulatory oxygen is unlikely to be effective at improving functional exercise capacity or symptoms of breathlessness in unselected patients with EID, although there may be some patients who respond well. Second, we have shown that use of OCDs is appropriate, in that outcomes are similar or better to CFNC but at lower oxygen consumption. Since the review showed no significant overall effect of oxygen, and there were no published cost-effectiveness studies, we were unable to calculate an incremental cost effectiveness ratio. Each area of the results will be discussed here in turn.

\section{Clinical Effectiveness of Ambulatory Oxygen}

The prescription of ambulatory oxygen therapy in COPD is governed by an improvement in exercise capacity or
Borg dyspnea scores, wherein a $10 \%$ improvement in distance walked or reduction $\geq 1$ in Borg Score indicates it should be used in patients with EID >4\%. ${ }^{5}$ The majority of our results suggest that there is not a benefit of this magnitude (Figures 2 and 3). It is important to highlight that in the included studies the definition of EID was not uniform, ranging from mild ( $<92 \%)$ to more severe desaturation $(<88 \%)$. Since results for some outcomes were heterogeneous it is important to consider how patient selection influenced results. Two studies ${ }^{12,18}$ used exertional dyspnea as their main inclusion criterion and were analyzed separately, although $1 / 3$ of patients in the Moore et al study also exhibited EID. Few studies specified acute responders (i.e., those with $>10 \%$ improvement in walking distance). The only study ${ }^{19}$ which did specify, used ESWT as the outcome measure and showed significant improvement in those randomized to ambulatory oxygen compared to compressed air, implying that appropriate patient selection is important in optimizing clinical outcome. 


\section{Figure 6. Effect of Oxygen Delivery Device on Exercise $\mathrm{SpO}_{2}$ and Exercise Capacity}

\begin{tabular}{|c|c|c|c|c|c|c|c|c|c|}
\hline \multirow[b]{2}{*}{ Study or Subgroup } & \multicolumn{3}{|c|}{ DODs } & \multicolumn{3}{|c|}{ CFNC } & \multicolumn{2}{|r|}{ Mean Difference } & \multirow{2}{*}{$\begin{array}{l}\text { Mean Difference } \\
\text { IV, Fixed, } 95 \% \mathrm{CI}\end{array}$} \\
\hline & Mean & SD & Total & Mean & SD & Total & Weight & IV, Fixed, $95 \% \mathrm{Cl}$ & \\
\hline Fuhrman 2004 & 260 & 93 & 13 & 257 & 93 & 13 & $0.7 \%$ & $3.00[-68.49,74.49]$ & \\
\hline Marti 2007 & 347.9 & 11.11 & 28 & 352.2 & 11.64 & 28 & $98.5 \%$ & $-4.30[-10.26,1.66]$ & \\
\hline Roberts 1996 & 283.3 & 88.8 & 15 & 295.4 & 89.1 & 15 & $0.9 \%$ & $-12.10[-75.76,51.56]$ & \\
\hline Total $(95 \% \mathrm{Cl})$ & & & 56 & & & 56 & $100.0 \%$ & $-4.32[-10.23,1.60]$ & \\
\hline $\begin{array}{l}\text { Heterogeneity: } \mathrm{Chi}^{2}= \\
\text { Test for overall effec }\end{array}$ & $\begin{array}{l}0.10, d f \\
Z=1.43\end{array}$ & $\begin{array}{l}=2(P= \\
(P=0.1\end{array}$ & $\begin{array}{l}0.95) ; \\
15)\end{array}$ & $1^{2}=0 \%$ & & & & & $\begin{array}{ccccc}-100 & -50 & 1 & 50 & 100 \\
\text { Favours [CFNC] } & \text { Favours [DODs] }\end{array}$ \\
\hline
\end{tabular}

$6.16 \mathrm{MWT}$

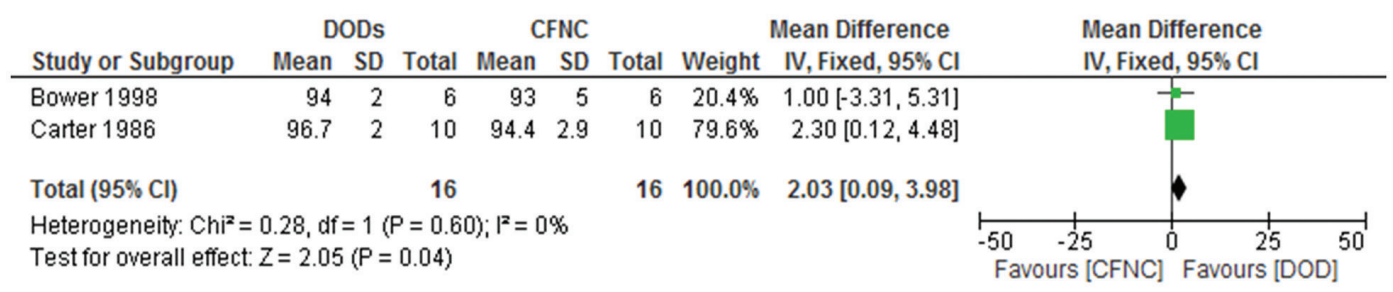

6.2 Exercise SpO2

Interestingly however, 7 studies ${ }^{12-16,18,20}$ included a single assessment acute oxygen test for all participants as part of their protocol. All but one ${ }^{20}$ demonstrated a significant mean improvement in exercise capacity for participants with acute oxygen therapy compared to compressed air which is in line with a previous Cochrane review. ${ }^{9}$ Whether the acute effects of oxygen persist in the long term in trained or untrained participants was addressed in 2 of the included studies ${ }^{13,14}$ as they all included a repeat acute oxygen test at the end of the study period. Rooyackers et $\mathrm{al}^{13}$ and Eaton et $\mathrm{al}^{14}$ both demonstrated that this acute effect was no longer statistically significant when using 6MWT as the outcome measure. It would therefore seem that any acute improvement in walking distance observed in a single assessment study is not maintained. This has implications for clinical practice as prescriptions for ambulatory oxygen based on improvements in exercise capacity observed in single assessment studies as suggested by guidelines ${ }^{5}$ are not sustained or are minimal in the longer term.

The way in which exercise capacity was measured may also be relevant to the interpretation of results. Largely it was measured using field tests (ESWT or $6 \mathrm{MWT}$ ) which, particularly in the case of the 6MWT, have been suggested to relate more to activities of daily living. ${ }^{35,36}$ The meta-analysis of exercise capacity with respect to $6 \mathrm{MWT}$ slightly favored the control group and showed no benefit of ambulatory oxygen, implying that in normal daily life it would not be expected to help patients be more active. In the ESWT meta-analysis, although the result was not statistically significant in favor of oxygen therapy, it may be clinically significant. The minimal clinically important difference (MCID) for the ESWT is estimated at $45-85$ seconds. ${ }^{37}$ The common effect of 150 seconds exceeded this although this has to be approached with caution because the heterogeneity between the 2 studies ${ }^{19,20}$ was considerable. Both studies enrolled patients exhibiting EID $>4 \%$ and predominantly severe COPD. During the ESWT in the Dyer et al study, patients had their oxygen cylinders carried by an assistant whereas this was not the case in Ringbaek et al. It is recognized that any improvement in exercise capacity can be negated by the weight of the ambulatory oxygen system if carried by patients alone, ${ }^{26,38}$ thus it is possible the Dyer study overinflated the benefits of treatment in real life by providing assistance. The meta-analysis of endurance cycle time strongly favored oxygen treatment and was close to approaching statistical significance. Furthermore, this is likely to be an underestimate of the effect of ambulatory oxygen treatment as 16 endurance tests in the oxygen group were terminated at 30 minutes compared to 6 endurance tests in the control group. ${ }^{15}$ The responsiveness of constant work rate tests such as the ESWT and cycle endurance to intervention is far better than the $6 \mathrm{MWT}^{39}$ which may explain the discrepancy between these different exercise 
outcome results. However, at present no MCID for cycle ergometry has been established and its relationship to activities of daily living is unclear.

Some studies ${ }^{13,15,17,19,20}$ were carried out as part of pulmonary rehabilitation. Although no statistical benefit of long term ambulatory oxygen was demonstrated, exercise capacity (particularly 6MWT) in the pulmonary rehabilitation studies exceeded that of the domiciliary studies ${ }^{12,18}$ whether patients were randomized to ambulatory oxygen or placebo, Figures 2.1 and 2.2 What is also clear is that the improvement in 6MWT distance gained by pulmonary rehabilitation far exceeds that gained by ambulatory oxygen. ${ }^{13}$ This supports current guidelines ${ }^{5}$ in that any assessment of ambulatory oxygen therapy should be made following pulmonary rehabilitation.

Finally, it is important to consider the meta-analyses possible using CRQ domains. In general, there was a benefit of ambulatory oxygen on QoL, which was most marked in the dyspnea and fatigue domains; the others were not statistically significant but showed a strong trend in the same direction. However, the effect size was only 0.28 and 0.17 in the statistically significant domains, which is less than the 0.5 points considered to represent the MCID for an individual domain. ${ }^{40}$ This explains the apparent discrepancy between Borg score and CRQ dyspnea score meta-analyses; neither were clinically significant, hence they concur, despite the fact that statistically the CRQ score was better with oxygen. The effect size is also considerably less than the effect on CRQ of pulmonary rehabilitation (0.62 points) ${ }^{41}$ confirming that assessment for ambulatory oxygen should only be done after rehabilitation.

\section{Impact of Device on Ambulatory Oxygen Delivery}

The mode of delivery of ambulatory oxygen therapy largely encompasses the cylinder type and the interface used. Oxygen conserving devices (DODs/PRC) have been used for many years and the theory supporting their use has been discussed elsewhere. ${ }^{23}$ In short classic DODs, devices deliver oxygen during the initial phase of inspiration alone while PRCs store oxygen during exhalation to be delivered at the next inspiratory phase. The role of OCDs is to conserve oxygen however, in doing so they must perform equivalently to standard CFNC in ameliorating oxygen desaturation. Some studies 22,24,26,30,34 included in the meta-analysis indicate that oxygen conserving devices perform equivalently to standard CFNC at maintaining exercise oxygen saturation and 6MWT. Importantly, however, it is likely that oxygen conserving devices achieve these outcomes at a much lower oxygen utilization than CFNC. This is likely to have implications in terms of oxygen cylinder usage and subsequent cost. Although the primary role of OCDs is not to improve exercise performance over CFNC, patients use these devices on the premise that they are ambulatory. Thus, it is reassuring that functional performance is not negated by the weight of the system.

It is important to note that there is considerable variation between OCDs and their performance and in the meta-analyses the best performing OCD was used. Notably in the study by Palwai et al, one of the DOD systems performed no better than ambulation on room air. Also there may be some patients who do not tolerate OCDs and their use may cause further desaturation. This is supported by the study by Marti et $\mathrm{al}^{34}$ in which use of DODs failed to correct EID in approximately $20 \%$ of participants. This has been explained by changing respiratory pattern on more strenuous exertion where by the predominance of mouth breathing rather than nasal fails to trigger the flow sensor. ${ }^{26}$ Oxygen cylinders have historically been the main method of delivering portable oxygen, however, newer devices such as portable concentrators are now available. These devices have variable weight with cylinders weighing between $3.4-8 \mathrm{~kg}$ and portable concentrators between $3-4 \mathrm{~kg}$. As mentioned previously, the weight of the ambulatory oxygen system (cylinder in this case) is an important factor as the portable oxygen system should be carried by the patient during their ambulatory oxygen assessment and, following this, in their ambulatory activity. The weight of the portable oxygen system has previously been cited as one of the reasons why patients with COPD have poor concordance with ambulatory oxygen prescriptions. ${ }^{42}$ An interesting observation from 1 of the studies ${ }^{33}$ is that ambulatory cylinder weight had no impact on home activity. One would hypothesize that a lower weight would facilitate ambulation. This was not the case despite prior efforts of focused education on increasing oxygen treatment understanding and ambulation.

Evidence from the included studies suggests that refilled oxygen cylinders/portable oxygen concentrators perform as well as standard commercial cylinders.

\section{Limitations}

The quality of data in this systematic review was 
poor to average as limited by small sample sizes and high risk of selection and detection bias, especially in the older studies. In addition, patient selection and heterogeneity of outcomes means that interpretation of the results should be guarded. Outcomes in this review did not include other clinically relevant endpoints such as exacerbations or mortality. Finally, there were no studies that assessed cost effectiveness. As clinical effectiveness could not be proven in any domain it was not possible to answer whether ambulatory oxygen is cost effective.

\section{Conclusions}

This review suggests that long term use of ambulatory oxygen has limited benefit compared to ambient air when assessed by functional exercise capacity or Borg dyspnea scores. It may, however, have greater efficacy in improving exercise performance when assessed by endurance tests. The impact on quality of life, although statistically significant, is unlikely to be clinically relevant. Where ambulatory oxygen is used oxygen conserving devices should be used, in preference to standard nasal cannula as they generally perform equivalently but at a lower oxygen utilization.

\section{Acknowledgements}

The authors would like to thank Dr. David Moore for assistance in designing the protocol. SE was the primary reviewer, conducted all statistical analysis and drafted the paper. AMT was second reviewer and reviewed the manuscript. SB conducted the literature searches. AG contributed to protocol design.

\section{Declaration of Interest}

SE is funded by Linde Real Fund and a non-commercial grant from CSL Behring. AG is funded by the West Midlands Chest Fund. AMT has received grants during the term of this work from the Linde Real fund, the NIHR and Alpha-1 Foundation. The most relevant of these for the submitted article is the Linde Real fund which was an unrestricted grant to conduct a clinical trial of ambulatory oxygen. She has also received payments or benefits of $<£ 10000$ over the last 5 years from several manufacturers of respiratory medicines, including GlaxoSmithKline, Novartis, Astra-Zeneca, Pfizer and Boehringer for educational talks and travel to meetings. The above funders had no role in data collection, statistical analysis or writing of this manuscript. SB has nothing to declare. 


\section{References}

1. Mathers CD, Loncar D. Projections of global mortality and burden of disease from 2002 to 2030. PLoS Med. 2006;3(11):e442. doi: http://dx.doi.org/10.1371/journal.pmed.0030442

2. Stoller JK, Panos RJ, Krachman S, Doherty DE, Make B. Oxygen therapy for patients with COPD: current evidence and the longterm oxygen treatment trial. Chest. 2010;138(1):179-187. doi: http://dx.doi.org/10.1378/chest.09-2555

3. Stolz D, Boersma W, Blasi F, et al. Exertional hypoxemia in stable COPD is common and predicted by circulating proadrenomedullin. Chest. 2014;146(2):328-338. doi: http://dx.doi.org/10.1378/chest.13-1967

4. Casanova C, Cote C, Marin JM, et al. Distance and oxygen desaturation during the 6-min walk test as predictors of long-term mortality in patients with COPD. Chest. 2008;134(4):746-752. doi: http://dx.doi.org/10.1378/chest.08-0520

5. British Thoracic Society. Clinical component for home oxygen services in England and Wales. British Thoracic Society website. https://www.brit-thoracic.org.uk/document-library/ clinical-information/oxygen/home-oxygen-guideline-(adults)/ bts-home-oxygen-in-adults-clinical-component/Published 2006. Accessed November 14, 2014.

6. American Thoracic Society/European Respiratory Society Task Force. Standards for the Diagnosis and Management of Patients with COPD 2004. American Thoracic Society website. http:// www.thoracic.org/copd-guidelines/. Published 2004. Accessed December 19, 2014.

7. Medical Research Council Working Party. Long term domiciliary oxygen therapy in chronic hypoxic cor pulmonale complicating chronic bronchitis and emphysema. Lancet. 1981:681-686.

8. Nocturnal Oxygen Therapy Trial Group. Continuous or nocturnal oxgen therapy in hypoxemic chronic lung disease: a clinical trial. Ann Intern Med. 1980; 93:391-398. doi: http://dx.doi.org/10.7326/0003-4819-93-3-391

9. Bradley JM, O'Neill B. Short term ambulatory oxygen for chronic obstructive pulmonary disease. Cochrane Database Syst Rev. 2005(2):CD004356.

10. Nonoyama ML, Brooks D, Lacasse Y, Guyatt GH, Goldstein RS. Oxygen therapy during exercise training in chronic obstructive pulmonary disease. Cochrane Database Syst Rev. 2007(2):CD005372.

doi: http://dx.doi.org/10.1002/14651858.cd005372.pub2

11. Jadad AR, Moore RA, Carroll D, et al. Assessing the quality of reports of randomized clinical trials: is blinding necessary? Control Clin Trials. 1996;17(1):1-12.

doi: http://dx.doi.org/10.1016/0197-2456(95)00134-4

12. McDonald CF, Blyth CM, Lazarus MD, Marschner I, Barter CE. Exertional oxygen of limited benefit in patients with chronic obstructive pulmonary disease and mild hypoxemia. Am J Respir Crit Care Med. 1995;152(5 Pt 1):1616-1619. doi: http://dx.doi.org/10.1164/ajrccm.152.5.7582304

13. Rooyackers JM, Dekhuijzen PN, Van Herwaarden CL, Folgering HT. Training with supplemental oxygen in patients with COPD and hypoxaemia at peak exercise. Eur Respir J. 1997;10(6):12781284. doi: http://dx.doi.org/10.1183/09031936.97.10061278
14. Eaton T, Garrett JE, Young P, et al. Ambulatory oxygen improves quality of life of COPD patients: a randomised controlled study. Eur Respir J. 2002;20(2):306-312. doi: http://dx.doi.org/10.1183/09031936.02.00301002

15. Emtner M, Porszasz J, Burns M, Somfay A, Casaburi R. Benefits of supplemental oxygen in exercise training in nonhypoxemic chronic obstructive pulmonary disease patients. Am J Respir Crit Care Med. 2003;168(9):1034-1042.

doi: http://dx.doi.org/10.1164/rccm.200212-1525OC

16. Nonoyama ML, Brooks D, Guyatt GH, Goldstein RS. Effect of oxygen on health quality of life in patients with chronic obstructive pulmonary disease with transient exertional hypoxemia. Am J Respir Crit Care Med. 2007;176(4):343-349. doi: http://dx.doi.org/10.1164/rccm.200702-3080C

17. Janaudis-Ferreira TH-LK, Lundgren R, Wadell K. Diferences in training effects following training with and without supplemental oxygen in patients with COPD. Adv Physiother. 2009;11:186-192. doi: http://dx.doi.org/10.3109/14038190802516704

18. Moore RP, Berlowitz DJ, Denehy L, et al. A randomised trial of domiciliary, ambulatory oxygen in patients with COPD and dyspnoeadyspnea but without resting hypoxaemia. Thorax. 2011;66(1):32-37. doi: http://dx.doi.org/10.1136/thx.2009.132522

19. Dyer F, Callaghan J, Cheema K, Bott J. Ambulatory oxygen improves the effectiveness of pulmonary rehabilitation in selected patients with chronic obstructive pulmonary disease. Chron Respir Dis. 2012;9(2):83-91.

doi: http://dx.doi.org/10.1177/1479972312438702

20. Ringbaek T, Martinez G, Lange P. The long-term effect of ambulatory oxygen in normoxaemic COPD patients: A randomised study. Chron Respir Dis. 2013;10(2):77-84. http://dx.doi.org/10.1177/1479972312473135

21. Soffer M, Tashkin DP, Shapiro BJ, Littner M, Harvey E, Farr S. Conservation of oxygen supply using a reservoir nasal cannula in hypoxemic patients at rest and during exercise. Chest. 1985;88(5):663-668. doi: http://dx.doi.org/10.1378/chest.88.5.663

22. Carter R, Williams JS, Berry J, Peavler M, Griner D, Tiep B. Evaluation of the pendant oxygen-conserving nasal cannula during exercise. Chest. 1986;89(6):806-810. doi: http://dx.doi.org/10.1378/chest.89.6.806

23. Tiep BL, Carter R, Nicotra B. Demand oxygen delivery during exercise. Chest. 1987;91(1):15-20. doi: http://dx.doi.org/10.1378/chest.91.1.15

24. Bower JS, Brook CJ, Zimmer K, Davis D. Performance of a demand oxygen saver system during rest, exercise, and sleep in hypoxemic patients. Chest. 1988;94(1):77-80. doi: http://dx.doi.org/10.1378/chest.94.1.77

25. Braun SR, Spratt G, Scott GC, Ellersieck M. Comparison of six oxygen delivery systems for COPD patients at rest and during exercise. Chest. 1992;102(3): 694-698.

26. Roberts CM, Bell J, Wedzicha JA. Comparison of the efficacy of a demand oxygen delivery system with continuous low flow oxygen in subjects with stable COPD and severe oxygen desaturation on walking. Thorax. 1996;51(8):831-834. doi: http://dx.doi.org/10.1136/thx.51.8.831 
27. Cuvelier A, Nuir JF, Chakroun N, Aboab J, Onea G, Benhamou D. Refillable oxygen cylinders may be an alternative for ambulatory oxygen therapy in COPD. Chest. 2002;122(2):451-456. doi: http://dx.doi.org/10.1378/chest.122.2.451

28. Tiep BL, Barnett J, Schiffman G, Sanchez O, Carter R. Maintaining oxygenation via demand oxygen delivery during rest and exercise. Respir Care. 2002;47(8):887-892.

29. Chatila W, Nugent T, Vance G, Gaughan J, Criner GJ. The effects of high-flow vs low-flow oxygen on exercise in advanced obstructive airways disease. Chest. 2004;126(4):1108-1115.

30. Fuhrman C, Chouaid C, Herigault R, Housset B, Adnot S. Comparison of four demand oxygen delivery systems at rest and during exercise for chronic obstructive pulmonary disease. Respir Med. 2004;98(10):938-944.

31. Strickland SL, Hogan TM, Hogan RG, Sohal HS, McKenzie WN, Petroski GF. A randomized multi-arm repeated-measures prospective study of several modalities of portable oxygen delivery during assessment of functional exercise capacity. Respir Care. 2009;54(3):344-349.

32. Palwai A, Skowronski M, Coreno A, Drummond C, McFadden ER. Critical comparisons of the clinical performance of oxygen conserving devices. Am J Respir Crit Care Med. 2010;181(10):1061-1071.

33. Casaburi R, Porszasz J, Hecht A, et al. Influence of lightweight ambulatory oxygen on oxygen use and activity patterns of COPD patients receiving long-term oxygen therapy. COPD. 2012;9(1):3-11.

doi: http://dx.doi.org/10.3109/15412555.2011.630048

34. Marti S, Pajares V, Morante F, et al. Are oxygen-conserving devices effective for correcting exercise hypoxemia? Respir Care. 2013;58(10):1606-1613.

doi: http://dx.doi.org/10.4187/respcare.02260

35. Guyatt GH, Thompson PJ, Berman LB, et al. How should we measure function in patients with chronic heart and lung disease? J Chron Dis. 1985;38(6):517-524.

doi: http://dx.doi.org/10.1016/0021-9681(85)90035-9

36. Redelmeier DA, Bayoumi AM, Goldstein RS, Guyatt GH. Interpreting small differences in functional status: the Six Minute Walk test in chronic lung disease patients. Am J Respir Crit Care Med. 1997;155(4):1278-1282.

doi: http://dx.doi.org/10.1164/ajrccm.155.4.9105067

37. Pepin V, Laviolette L, Brouillard C, et al. Significance of changes in endurance shuttle walking performance. Thorax. 2011;66(2):115120. doi: http://dx.doi.org/10.1136/thx.2010.146159

38. Leggett RJ, Flenley DC. Portable oxygen and exercise tolerance in patients with chronic hypoxic cor pulmonale. Brit Med J. 1977;2(6079):84-86. http://dx.doi.org/10.1136/bmj.2.6079.84

39. Borel B, Provencher S, Saey D, Maltais F. Responsiveness of Various Exercise-Testing Protocols to Therapeutic Interventions in COPD. Pulm Med. 2013;2013:410748. doi: http://dx.doi.org/10.1155/2013/410748

40. Schunemann HJ, Puhan M, Goldstein R, Jaeschke R, Guyatt GH. Measurement properties and interpretability of the Chronic Respiratory Disease Questionnaire (CRQ). COPD. 2005;2(1):81-89.

doi: http://dx.doi.org/10.1081/COPD-200050651
41. Kon SS, Dilaver D, Mittal M, et al. The Clinical COPD Questionnaire: response to pulmonary rehabilitation and minimal clinically important difference. Thorax. 2014;69(9):793798. doi: http://dx.doi.org/10.1136/thoraxjnl-2013-204119

42. Arnold E, Bruton A, Donovan-Hall M, Fenwick A, Dibb B, Walker E. Ambulatory oxygen: why do COPD patients not use their portable systems as prescribed? A qualitative study. BMC Pulm Med. 2011;11:9. doi: http://dx.doi.org/10.1186/1471-2466-11-9 